Journal of Electronics and Informatics (2020)

Vol.02/ No. 02

Pages: 80-87

http://www.irojournals.com/iroei/

DOI: https://doi.org/10.36548/jei.2020.2.002

\title{
Data Forwarding in Wireless Body Area Networks
}

\author{
Dr. Joy Iong Zong Chen, \\ Professor, \\ Department of Electrical Engineering, \\ Da-Yeh University,Taiwan. \\ Email id: jchen@mail.dyu.edu.tw \\ Lu-Tsou Yeh, \\ Department of Electrical Engineering, \\ Da-Yeh University, \\ No. 168 University Rd., \\ Dacun, Changhua 51591, \\ Taiwan
}

\begin{abstract}
One of the most crucial application of Wireless Body Area Networks in healthcare applications is the process of monitoring human bodies and gather physiological data. Network performance degradation in the form of energy efficiency and latency are caused because of energy depletions which arises due to limited energy resource availability. The heterogeneity of body sensors will lead to variation in the rate of energy consumption. Based on this, a novel Data Forwarding Strategy is presented in this research work to enhance collaborative WBAN operations, improve network lifetime and restrict energy consumption of the sensors. In this paper, we have contributed towards reducing the size of data to be transmitted by compressed sensing and selection of relay sensor based on sampling frequency, energy levels and sensor importance. Using the proposed methodology, it is possible to improve both reliability and energy-efficiency of WBAN data transmission. moreover, it is also possible to adapt to the changing WBAN topologies when the proposed methodology is used, balancing energy efficiency and consumption.
\end{abstract}

Keywords: WBAN, network performance, data forwarding, human bodies, balance energy consumption

\section{Introduction}

Wireless Body Area Networks (WBANs) are a special type of Wireless Sensor Networks that are used for healthcare and biochemical areas[1]. In the recent years, they have gained much attention from the government, industries [2] and academia [3]. The WBAN comprises of body sensors with monitoring and processing capabilities that can be implanted or worn in order to gather physiological information such as electrocardiogram, blood pressure [4], blood oxygen levels, pulse [6], temperature, electroencephalogram etc. However, in WBAN, there is limitation on resources and energy availability of sensors. Moreover, sensors which are implanted are complex [5] and costly. In order to increase the efficiency of energy as well as to improve the lifespan of WBANs, many research have been carried out. In general, the WBANs are quite small in size and based on the amount of data transmitted by them along with the choice of relay sensors, the total amount of energy consumed during the process of data 
Journal of Electronics and Informatics (2020)

Vol.02/ No. 02

Pages: 80-87

http://www.irojournals.com/iroei/

DOI: https://doi.org/10.36548/jei.2020.2.002

forwarding is determined. Based on some of the existing work on energy consumption design and sensor structures, one of the biggest contributor of sensor energy consumption is data transmission. Hence by decreasing the data size it is possible to improve the efficiency of sensor energy efficiency. In the realworld scenario, the WBANs used for health monitoring applications are affected by shadow effects formed due to body movements. This will result in changing the network topology as well as the wireless link status resulting in complications of the WBANs, leading to challenges in proper monitoring of the human physiological parameters. This has made for infeasibility of the traditional routing mechanisms. In [7] and [8] researchers have developed the process of transmitting data using multi-hop methodology with the use of relay nodes which will improve the robustness of the sensor communication system. However, enhanced data delivery ratio coupled with reduction in consumption of energy is possible when the proposed multi-hop data forwarding system is employed and simulation results support this theory.

The major contribution of this research work is as follows:

- The proposed data forwarding structure collaboratively exploits the use of energy resource in order to prevent unnecessary power consumption by other sensors resulting in the formation of a robust WBAN with changing topologies.

- In order to balance network lifetime and energy consumption of sensors, sensor importance degree, residual energy and sampling frequency are collaboratively used to select the best relay sensor.

- The data collected through physiological contact is compressed using CS technology and energy consumption also reduces simultaneously.

\section{System Model}

Tree multi-hop network topologies and star single hop topologies are the most commonly used WBANs [9]. In the single-hop network, physiological data is gathered by the sink using body sensors, This is then forwarded to a central unit from where the feedback for the received data is sent back to the body sensors. This process of data transmission in bi-directional way is accomplished in a single-hop fashion. Similarly star topology is also used gather data and give real-time data through closer inter-node distance between the small networks of WBANs [10-11]. The drawback with star topology is that it will result in unwanted heat emission, higher energy consumption and high transmission power of sensor because of packet loss and high level of accuracy. In order to overcome these shortcomings, a multi-hop tree topology is formulated which has eventually become popular for its optimal usage of body sensors by forwarding the gathered data to body sensors far away. As a result of this effect, remote body sensors consume lesser transmission power [12] while balancing network energy consumption to ensure data transmission in a reliable fashion. 
Journal of Electronics and Informatics (2020)

Vol.02/ No. 02

Pages: $80-87$

http://www.irojournals.com/iroei/

DOI: https://doi.org/10.36548/jei.2020.2.002

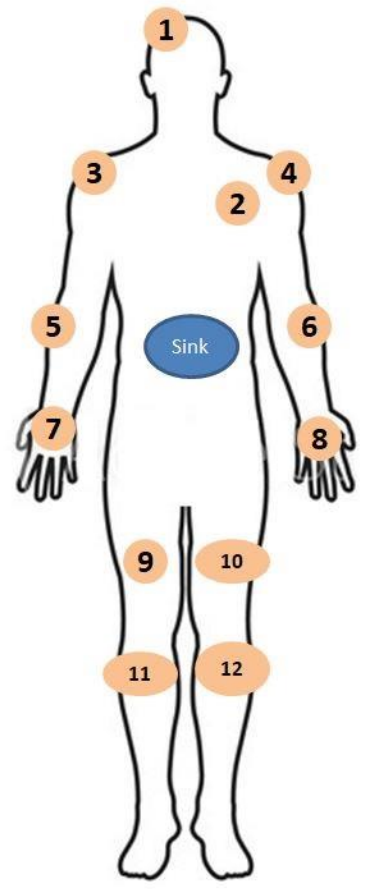

Fig.1 System Structure

In a WBAN network, an energy efficient data forwarding system plays an important role. Consider the system structure in Fig.1. which outlines specific application targets that can be utilized to transmit data. Here multiple body sensors are used to gather data from the body and forward this information to the sink. The transmission process takes place within many hops and the remote body sensors need to monitor and guide the data by forwarding it via the right relay. There are three major components of energy consumption in equation 1 ,

$$
E_{\text {total }}=E_{t}+E_{r}+E_{p}
$$

where $E_{t}, E_{r}$ and $E_{p}$ are the energy consumed for transmitting, receiving and processing data packets and $E_{\text {total }}$ represents the total energy consumed.

If $\mathrm{d}$ is the transmission distance, $\mathrm{n}$ is the length of data packet, $E_{a m p}$ is the amplyfying circuit's energy consumption, $E_{e}$ is the energy consumed for transmission of data bit and $\alpha$ is the transmission power loss coefficient, then

$$
\left\{\begin{array}{c}
E_{r}=n * E_{e} \\
E_{t}=n *\left(E_{e}+E_{a m p} d^{\alpha}\right.
\end{array}\right.
$$

ISSN: 2582-418X (online) 
Journal of Electronics and Informatics (2020)

Vol.02/ No. 02

Pages: 80-87

http://www.irojournals.com/iroei/

DOI: https://doi.org/10.36548/jei.2020.2.002

The cost of employing CS which is inclusive of energy consumed for data processing is significantly high. Hence the total energy consumed can be calculated as

$$
E_{\text {Total }}=n *\left(2 E_{e}+E_{a m p} d^{\alpha}\right)+E_{p}
$$

\section{Compression of Data}

The real-time monitoring of a human physiology will result in generation of data in large quantities. However, both processing capability as well as energy resource of WBANs sensors are highly constrained. Hence, the transmission of this large quantity of data will lead to network congestion and increased energy consumption. In order to tackle this issue, the physiological data which is gathered is initially compressed using CS at the sensors. This is accomplished by using sampling frequencies which are very low when compared with Nyquist frequency, resulting in the data being represented as sparse vectors which have reduction of data size. This will also improve the energy consumed by the network. The data thus obtained can be reversed back to its original form using the sink.

The data collected in this research work is represented as $y=y_{1}, y_{2}, y_{3}, \ldots \ldots ., y_{N}$ with the basic vector being represented as $\psi_{\mathrm{i}}$ such that $\psi$ is the sparse basis and $\alpha$ is the coefficient vector representing $\alpha=\left[\alpha^{1}, \alpha^{2}, \ldots \ldots, \alpha^{N}\right]^{\mathrm{T}}$

$$
Y_{N}^{T} * 1=\sum_{i=1}^{N} a_{i} \psi_{\mathrm{i}}=\psi_{N} * N^{\alpha}
$$

Here $\mathrm{Y}$ can used to compress the data to represent it in a $\mathrm{M}$ dimensional measurement data that can be represented in the equation below.

$$
Z_{m * 1}=\theta_{M * N} \times Y_{N}^{T} * 1=\theta \times \psi \times \alpha
$$

On receiving the physiological data from the sensors, there are two challenges faced. The received data is first used to develop a measurement matrix by comparing the original data set with the preset sparse binary data set. Next is the process of redeveloping the original data from the compressed data. The first condition that the received data should satisfy is that it should be independent of $\psi$. Toeplitz, Hadamard, Gaussian random, random Fourier and Bernoulli random matrices are some of the methodologies used for this purpose in CS technology. However, to simplify the process as well as to reduce difficulty in implementation, the technology used in this paper is sparse binary random 
Journal of Electronics and Informatics (2020)

Vol.02/ No. 02

Pages: $80-87$

http://www.irojournals.com/iroei/

DOI: https://doi.org/10.36548/jei.2020.2.002

measurement matrix. Here the proposed methodology can convert a complex matrix multiplication into that of addition, thereby decreases the amount of energy consumed drastically.

\section{Relay Sensor Selection}

Research shows that several hops are involved in transmitting the data packets to the sink. Hence the choice of relay sensors plays a major role in affecting the performance of the system as well as the energy consumption. The choice of optimal relay sensor is done by considering the rate at which energy is consumed and the residual energy level of the sensor. In general, there is a difference in the rate at which energy is consumed by the different sensors. A good example is that the transmission of data from sensors that are monitoring body temperature, blood oxygen level etc will require lesser energy consumption when compared with sensors that are used to measure ECG signals. Hence it is necessary to that sensors which require high energy should be preserved and their forwarding should be reduced in order to control and decrease their energy consumption.

\section{Simulation Results}

In star topology 4 sensor nodes are selected and the energy consumed are shown in the simulation Fig.2.

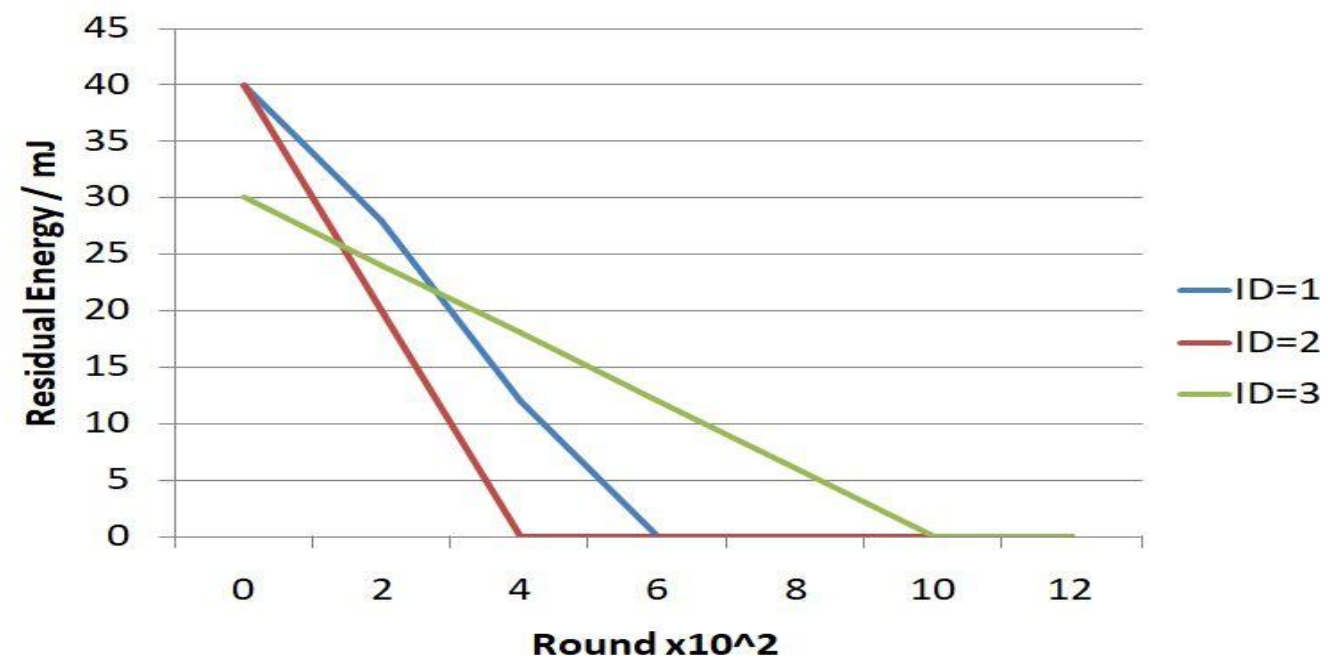

Fig.2. Energy consumption in different postures for star network topology

In the proposed data forwarding algorithm, there is a relativity between network running time and energy consumption which is depicted through simulation in Fig. 3. It can be observed that the residual energy for different postures eventually approach zero as the round count increases. As the proposed 
Journal of Electronics and Informatics (2020)

Vol.02/ No. 02

Pages: $80-87$

http://www.irojournals.com/iroei/

DOI: https://doi.org/10.36548/jei.2020.2.002

methodology uses uniform relay sensor selecting, for various body postures, the ECG signal is found to be similar.

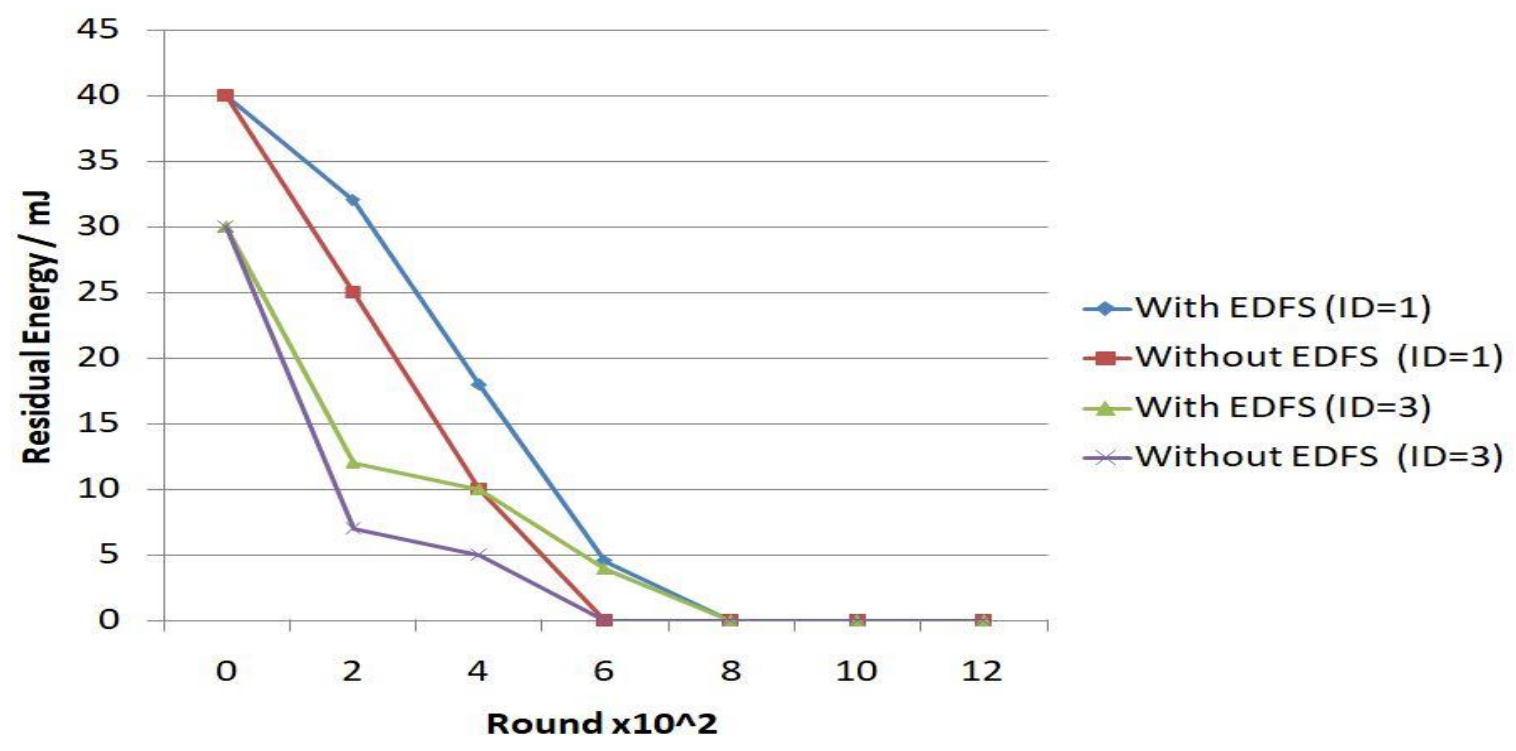

Fig.3. Energy consumption with and without data forwarding

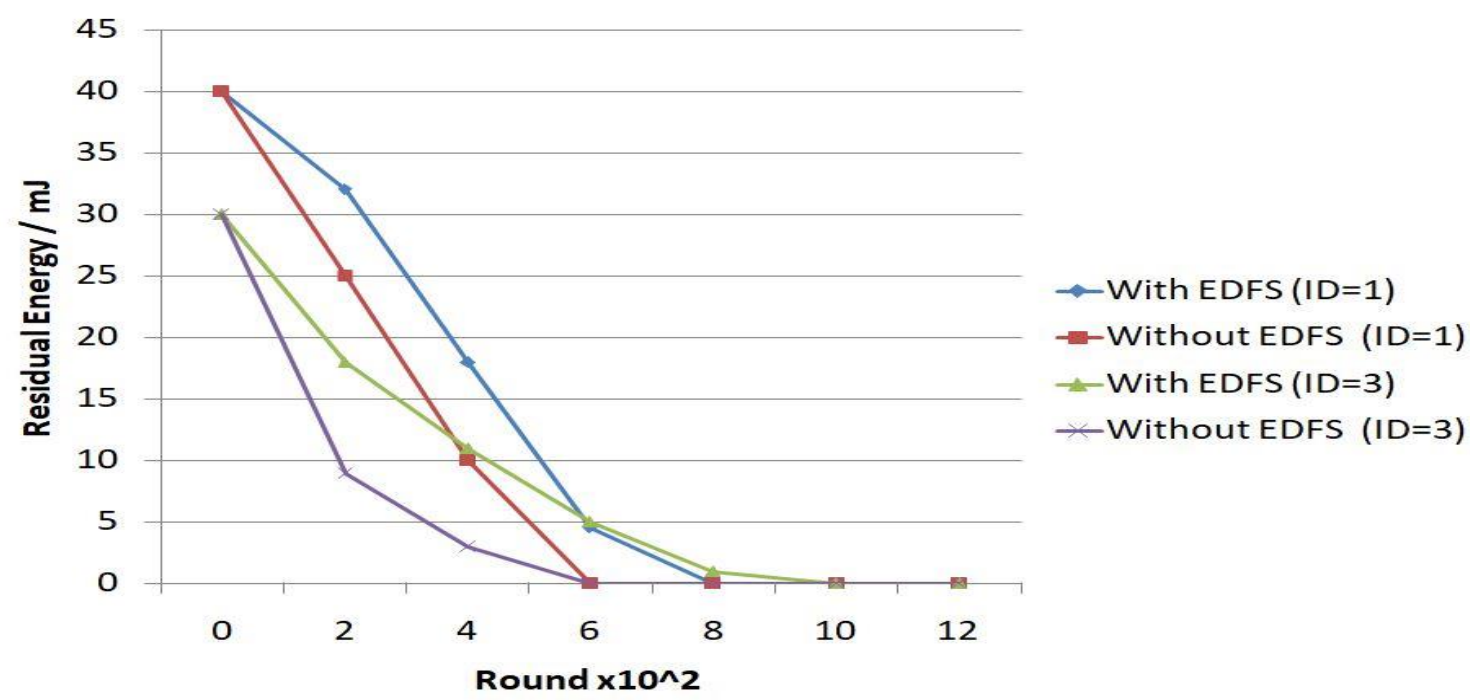

Fig.4. Energy consumption with and without data forwarding

When comparing the different energy consumed by different postures, it is found that the overall lifetime of the various sensors which collect ECG and EEG signals can be increased and improved. Moreover, as the relays are selected, it will also further decrease the network topology. 
Journal of Electronics and Informatics (2020)

Vol.02/ No. 02

Pages: $80-87$

http://www.irojournals.com/iroei/

DOI: https://doi.org/10.36548/jei.2020.2.002

\section{Conclusion}

Because of WBAN sensor's restriction on energy resources, high energy consumption will have a great impact on the performance of the system, thereby degrading it. Hence the proposed work focuses on improving the network lifetime while balancing the amount of energy used by the sensors of the WBANs. The actual data is processed using CS technology to compress the size of physiological data and then using the optimal relay choice, it is possible to obtain a good composition of efficiency and reliability of the network. In the future, the same can be expanded to harvesting of energy and the methodologies used during this process can be determined.

\section{References}

[1] Verbiest, J. R., \& Vandenbosch, G. A. (2006). A novel small-size printed tapered monopole antenna for UWB WBAN. IEEE Antennas and Wireless Propagation Letters, 5, 377-379.

[2] J. Naganawa, K. Wangchuk, M. Kim, T. Aoyagi, and J.-I. Takada, 'Simulation-based scenario-specific channel modeling for WBAN cooperative transmission schemes," IEEE J. Biomed. Health Informat., vol. 19 no. 2, pp. 559_570, Mar. 2015.

[3] M. Balouchestani, K. Raahemifar, and S. Krishnan, "Wireless body area networks with compressed sensing theory," in Proc. ICME Int. Conf. Complex Med. Eng. (CME), Jul. 2012 pp. 364_369.

[4] L. Feng et al., "Golden-angle radial sparse parallel MRI: Combination of compressed sensing, parallel imaging, and golden-angle radial sampling for fast and _exible dynamic volumetric MRI," Magn. Reson. Med., vol. 72, no. 3, pp. 707_717, 2014.

[5] D. Gangopadhyay, E. G. Allstot, A. M. R. Dixon, K. Natarajan, S. Gupta, and D. J. Allstot, "Compressed sensing analog front-end for bio-sensor applications," IEEE J. Solid-State Circuits, vol. 49, no. 2, pp. 426_438, Feb. 2014.

[6] F. Chen, F. Lim, O. Abari, A. Chandrakasan, and V. Stojanovic, “Energyaware design of compressed sensing systems for wireless sensors under performance and reliability constraints," IEEE Trans. Circuits Syst. I, Reg. Papers, vol. 60, no. 3, pp. 650_661, Mar. 2013.

[7] S. Yang, J. L. Lu, F. Yang, L. Kong, W. Shu, and M. Y. Wu, "Poster: Behavior-aware probabilistic routing for wireless body area sensor networks," in Proc. IEEE GLOBECOM, Apr. 2013, pp. 444_449.

[8] Devana, V. K. R., \& Rao, A. M. A Compact 3.1-18.8 GHz Triple Band Notched UWB Antenna for mobileUWB Applications. IRO Journal on Sustainable Wireless Systems (2020) Vol.02/ No. 1 Pages: 1- 12

[9] L. Liang, Y. Ge, G. Feng, W. Ni, and A. A. P. Wai, “'A low overhead treebased energyef_cient routing scheme for multi-hop wireless body area networks," Comput. Netw., vol. 70, no. 18, pp. 45_58, 2014. 
Journal of Electronics and Informatics (2020)

Vol.02/ No. 02

Pages: $80-87$

http://www.irojournals.com/iroei/

DOI: https://doi.org/10.36548/jei.2020.2.002

[10] Li, C., Li, H. B., \& Kohno, R. (2009, June). Performance evaluation of IEEE 802.15. 4 for wireless body area network (WBAN). In 2009 IEEE International conference on communications workshops (pp. 1-5). IEEE.

[11] X. Zhang, H. Wu, and Y. Ma, “A new auto-focus measure based on medium frequency discrete cosine transform filtering and discrete cosine transform," Appl. Comput. Harmon. Anal., vol. 40, no. 2, pp. 430_437, 2016

[12] Bashar, D. A. (2020). Review on sustainable green Internet of Things and its application. J. Sustain. Wireless Syst., 1(4), 256-264

\section{Authors Biography}

Dr. Joy Iong-Zong Chen is currently a full professor of Department of Electrical Engineering Dayeh University at Changhua Taiwan. Prior to joining the Dayeh University, he worked at the Control Data Company (Taiwan) as a technical manager since Sep. 1985 toSep. 1996. His research interests include wireless communications, spread spectrum technical, OFDM systems, and wireless sensor networks. He has published a large number of SCI Journal papers in theissues addressed physical layer for wireless communication systems. Moreover, he also majors in developing some applications of the IOT (Internet of Thing) techniques and Dr. Joy I.-Z. Chen owned some patents authorized by the Taiwan Intellectual Property Office (TIPO)

Lu-Tsou Yeh, works as professor in Department of Electrical Engineering, Da-Yeh University, No. 168 University Rd., Dacun, Changhua 51591, Taiwan, his major area of research aresemiconductor materials, computer science, nano electronics, object/web technologies, microelectronics, quantum electronics, VLSI, electronic system design, IT integrated manufacturing, fabrication, and analysis which remains as the backbone for developing nextgeneration electronic devices and information technology applications 